\title{
General Stress Conditions Dominating in the Hydride Precipitation Zone of Metals Under Steady State
}

\author{
A.G. Varias* \\ Ministry of Defense, Hellenic Navy, Athens, Greece

\begin{abstract}
The general stress conditions, which dominate in the zone of hydride precipitation under steady state for hydrogen diffusion, are derived, by rigorously taking into account the coupling of the operating physical processes. The conditions are valid, irrespective of metal elastic anisotropy, plastic deformation or geometry. According to these conditions, stress trace is a function only of temperature, leading to loss of dominance of the well-known asymptotic crack-tip stress fields of hydrogen-free metals, including those of constraint-based fracture mechanics. The present analysis is applied to a hydrided cylindrical wall and a hydrided plate under steady state heat conduction. It is shown that a nearly linear variation of hydrostatic stress dominates within the area of hydride precipitation, even in the presence of a surface crack, being in excellent agreement with existing detailed finite element calculations.
\end{abstract}

Keywords: Hydride precipitation, hydrogen, hydrostatic stress, stress trace.

\section{INTRODUCTION}

The defect assessment procedures, widely used in engineering, are based on asymptotic crack tip fields of linear elastic or non-linear material behavior. It is assumed, that, in a solid with a crack, an annulus exists, around the crack tip, where stress and deformation fields have the structure of the asymptotic crack tip fields. As a result, the fracture process and therefore material toughness is characterized by the parameters of the dominating asymptotic fields.

It is well known, that, in elastic-plastic materials under small scale yielding, there is an annulus, where the leading term of the elastic crack-tip field asymptotic expansion dominates [1]. Then the stress intensity factor, $K$, or the energy release rate, $G$, is the characteristic parameter and provides material fracture toughness. When the conditions of small scale yielding are violated, $J$-integral [2] and the associated HRR crack-tip field [3, 4] form the basis for a oneparameter fracture analysis. HRR field describes the deformation in a region around the crack tip within the plastic zone, if certain conditions are satisfied, which depend on material properties, geometry and type of loading (e.g. [5]).

The demand for extending the life of old structures has led to the development of constraint based fracture mechanics, outside the regime of applicability of one-parameter approaches (e.g. [6-8]). According to constraint based fracture mechanics, there exist a one-parameter family of elasticplastic field distributions. Members of this family describe the near-tip distributions of cracked geometries, under various loading conditions and amounts of yielding. Under contained yielding, T-stress, the amplitude of the second term of Williams' expansion [1], can serve as a parameter of the

*Address correspondence to this author at the Ministry of Defense, Hellenic Navy, Athens, Greece; Tel: +30-210-2821655; Fax: +30-210-2849916; E-mail: smro@hol.gr family of distributions. Another parameter, which is appropriate also for full scale yielding, is Q, a hydrostatic stress coefficient of the second term in the elastic-plastic asymptotic expansion of the crack tip field [7]. Variation of T or Q leads to crack-tip stress distributions with different levels of triaxiality, which is equal to the ratio of hydrostatic over yield stress, and consequently leads to different conditions of material degradation. Then, according to constraint based fracture mechanics, instead of a single value for fracture toughness, there is a toughness locus, which is a function of T or Q.

Fracture mechanics approaches, based on the asymptotic crack tip fields, were also applied to analyze sub-critical crack growth in hydride forming metals, also called delayed hydride cracking. The experimental investigations revealed a threshold of crack growth initiation as well as a region of steady crack growth, caused by brittle hydride precipitation (e.g. [9-12]). Parallel to the experimental studies, analytical models were developed for predicting steady-state crack growth velocities (e.g. [13, 14]). These models assumed the dominance of near-tip elastic or elastic-plastic fields of hydrogen-free metals, within the area of hydride precipitation. It is worth mentioning that hydride-induced embrittlement and sub-critical crack growth are complicated mechanisms, which result from the simultaneous operation of several coupled processes, namely, (i) hydrogen diffusion, (ii) hydride precipitation, (iii) non-mechanical energy flow, including heat conduction, and (iv) material deformation (e.g. [15]). Indeed, hydrogen diffusion is driven by the gradients of stress, temperature and hydrogen concentration. Hydride precipitation depends on temperature as well as on stress, due to hydride transformation strain. Finally, material deformation is affected by volume changes, caused by hydrogen dissolution, hydride formation and temperature variation. Motivated by the effects of the coupled processes, one could question any implicit assumption for the existence of 
an annulus, where an asymptotic crack tip field, developed for a hydrogen-free metal, dominates, and insist on a confirmation. At no surprise, detailed finite element studies [1517], which took into account the coupling of the operating physical processes, revealed that the well-known asymptotic crack tip fields, based on linear elastic or elastic-plastic or even on constraint based fracture mechanics, are not valid within the zone of hydride precipitation. In [15] the experimentally observed discontinuous process of sub-critical crack growth was simulated, while in [17], the theoretical predictions of steady-state crack growth velocity found to be in agreement with experimental measurements and reproduced the effects of temperature and yield stress.

The objective of this paper is to develop analytically and present the stress conditions, which dominate within the hydride precipitation zone and lead to the deviation from the well-known asymptotic crack-tip fields. It is shown that these conditions do not depend on deformation properties, elastic or elastic-plastic, of solid solution and hydrides. Also they do not depend on hydride size, shape or orientation. Consequently, the present work provides the basis for future investigation of the conditions, under which an annulus of dominance of an asymptotic crack-tip field exists in a cracked structure, when hydride precipitation occurs. Such investigations are required for the application of fracture mechanics in structural integrity assessments, when hydrideinduced fracture is expected.

The present analysis assumes that there is sufficient time for redistribution of hydrogen, establishing steady state conditions and approach of zero hydrogen flux in the zone of hydride precipitation. These conditions develop ahead of a crack tip, before the initiation of delayed hydride cracking as well as during crack propagation near the threshold $[15,16]$.

\section{GENERAL STRESS CONDITIONS IN THE HYDRIDE PRECIPITATION ZONE}

Let us consider the zone of hydride precipitation in a metal, under the presence of heat flux and mechanical loading. It is assumed that hydrides do not form a continuous network and therefore the redistribution of hydrogen, within the solid solution, is not inhibited.

The diffusion of hydrogen in the solid solution depends on the distributions of hydrogen concentration, stress and temperature, according to the following relation (e.g. [15, 18]), which is written in a Cartesian coordinate system $\left(x_{1}, x_{2}, x_{3}\right)$ :

$J_{k}^{H}=-D^{H} \frac{\partial C^{H}}{\partial x_{k}}+\frac{D^{H} C^{H} \bar{V}^{H}}{3 R T} \frac{\partial \sigma_{m m}}{\partial x_{k}}-\frac{D^{H} C^{H} Q^{H}}{R T^{2}} \frac{\partial T}{\partial x_{k}}$

In (1) as well as in following relations, Latin indices, indicating the components of vectors and second or higher order tensors, range from one to three; repeated Latin indices are always summed. $J_{k}^{H}, C^{H}, \sigma_{m m}$ and $T$ are the components of hydrogen flux, hydrogen concentration, stress trace and absolute temperature in the solid solution, respectively. $R\left(=8.314 \mathrm{~J} \mathrm{~mol}^{-1} \mathrm{~K}^{-1}\right)$ is the gas constant. Also $\bar{V}^{H}, D^{H}$ and $Q^{H}$ are the molal volume, the diffusion coefficient and the heat of transport of hydrogen in the solid solution. According to the mathematical description of hydrogen diffusion, discussed in detail in $[15,19]$, which is based on the thermody- namic theory of stressed solids [20] and irreversible processes [21], in relation (1), the gradient of each driving physical quantity, i.e. of $C^{H}, \sigma_{m m}$ and $T$, is derived at constant values of the other quantities.

Hydride precipitation is assumed to occur under local chemical equilibrium among hydrogen, metal and hydride. Then hydrogen concentration in the solid solution of the hydride precipitation zone is equal to the terminal solid solubility, given by the following general relation [16]:

$$
\begin{aligned}
& C^{T S}=C_{e}^{T S} \exp \left(\frac{\bar{w}_{\text {int }}}{x R T}\right) \exp \left(\frac{\sigma_{k k} \bar{V}^{H}}{3 R T}\right) \\
& \bar{w}_{\text {int }}=-\int_{\bar{V}^{h r}} \sigma_{m n}^{h} \varepsilon_{m n}^{T} d V \\
& -\frac{1}{2} \int_{\bar{V}^{h r}}\left(D_{i j k l}^{M}-D_{i j k l}^{h r}\right)\left(D_{k l r s}^{M}\right)^{-1}\left(D_{i j p q}^{h r}\right)^{-1} \sigma_{r s} \sigma_{p q}^{h} d V
\end{aligned}
$$

In (2a)-(2b), $\varepsilon_{i j}^{T}$ is the stress-free transformation strain of a precipitating hydride. $C_{e}^{T S}$ is the terminal solid solubility of hydrogen, under no applied stress, and incorporates the strain energy, which is required for the accommodation of the expanding hydride. $C_{e}^{T S}$ is derived experimentally and it is an exponential function of temperature (e.g. [18]). $\bar{w}_{\text {int }}$ is the interaction energy per mole of precipitating hydride of volume $\bar{V}^{h r}$, which results from the interaction of the applied stress field, $\sigma_{i j}$, with the field of the expanding hydride, as well as from the change of the elastic material properties, when phase transformation occurs. $D_{i j k l}^{M}$ and $D_{i j k l}^{h r}$ are the elastic stiffness of the metal and the hydride, respectively. $\sigma_{i j}^{h}$ is the stress field in the hydride, under the applied stress, $\sigma_{i j}$, when no hydride expansion occurs. Finally, $x$ is the mole fraction of hydrogen in the hydride, which is generally of type $\mathrm{MH}_{x}$. Relations (2a) and (2b) are valid for hydrides of any shape. Metallic and hydride phases may also have any degree of elastic anisotropy. The above relations are simplified, when it is assumed that hydride and metal have the same elastic properties. In this case and under hydride volumetric expansion, $\varepsilon^{h r}\left(=\varepsilon_{k k}^{T}\right)$, the interaction energy takes the form:

$\bar{w}_{\text {int }}=-\frac{\sigma_{k k}}{3} \varepsilon^{h r} \bar{V}^{h r}$

According to (2a) and (2b), i.e. even in the most general case, at constant stress and temperature, hydrogen terminal solid solubility and, therefore, hydrogen concentration in the solid solution of the hydride precipitation zone is constant. Consequently, when steady state, with respect to hydrogen diffusion, is established and hydrogen flux equals to zero, (1) and (2) lead to the following relation of stress trace gradient:

$\frac{\partial \sigma_{m m}}{\partial x_{k}}=\frac{3 Q^{H}}{\bar{V}^{H}} \frac{1}{T} \frac{\partial T}{\partial x_{k}}$

Integration of (4) provides a general stress condition, which is valid in the solid solution of the hydride precipitation zone: 


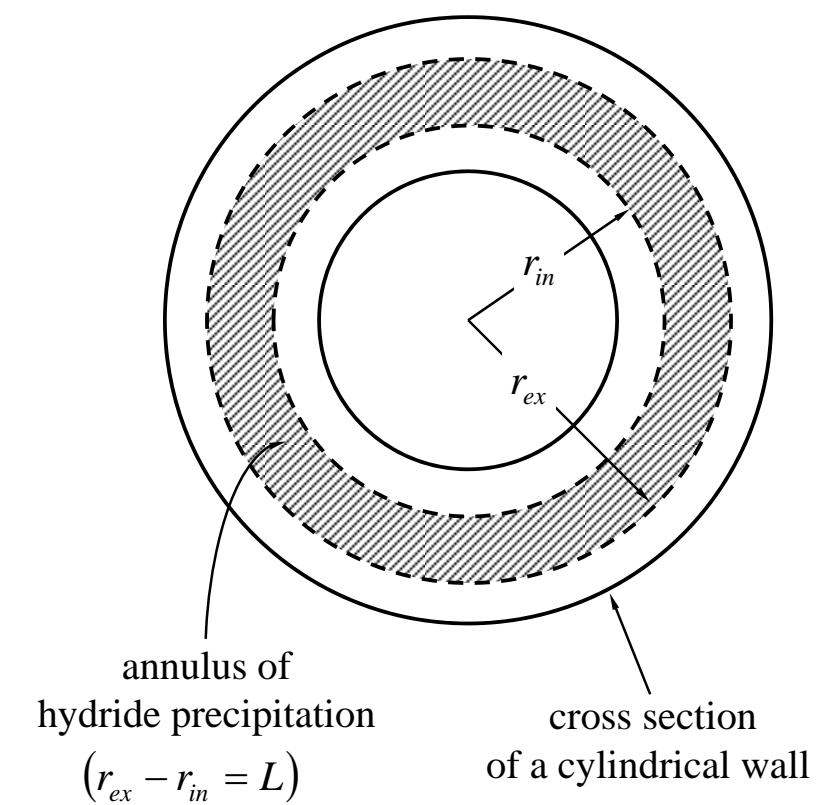

Fig. (1). Structure and geometrical parameters of an infinitely long hydrided cylinder.

$-\frac{\bar{V}^{H}}{3} \sigma_{m m}+Q^{H} \ln T=$ const.

According to (4) and (5), the stress trace and its gradient depend only on temperature distribution. Relations (4) and (5) are valid, irrespective of phase mechanical properties and deformation. They are also valid, irrespective of size, shape or orientation of the hydrides within the precipitation zone. It is emphasized that, the presence of geometric irregularities or discontinuities, such as cracks, corners or holes, does not invalidate (4) and (5).

Under constant temperature ( $\left.T=T_{0}\right)$, relation (5) requires a constant value of stress trace and consequently of hydrostatic stress:

$$
\sigma_{m m}=\text { const. }, T=T_{0}
$$

Indeed, the detailed finite element calculations, presented in [16], have shown that a constant hydrostatic stress dominates in the hydride precipitation zone around a crack tip, when the crack propagates steadily, under constant temperature, near the threshold stress intensity factor. The calculations were performed for Zircaloy- 2 and $\delta$-hydride $\left(\mathrm{ZrH}_{1.66}\right)$ and took into account material's plastic deformation as well as different elastic properties of metallic and hydride phases.

Relations (4) to (6) are rigorous, presenting general limits, as steady state for hydrogen diffusion is approached, and valid within the solid solution of the hydride precipitation zone. Indeed, when there are no precipitated hydrides, steady state may be reached at varying hydrogen concentration, in which case relations (4) to (6) do not apply. Also, if, for mathematical simplicity, the presence of the hydrides is described by hydride volume fraction, i.e. the hydrides are smeared, the above relations could be applied throughout the hydride precipitation zone. In this case, the hydride precipitation zone is treated as a composite, made of solid solution and hydrides, with effective material properties.

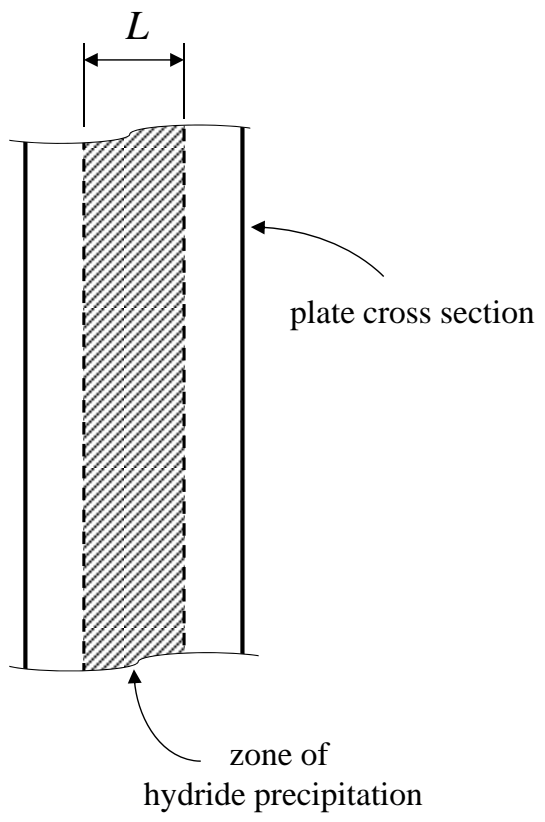

Fig. (2). Structure and geometrical parameters of a hydrided infinite plate.

In the following section, emphasis is placed on the stress conditions, which dominate in the hydride precipitation zone under temperature variations, i.e. in the presence of heat flux.

\section{DISTRIBUTION OF HYDROSTATIC STRESS IN HYDRIDED CYLINDER AND PLATE UNDER STEADY STATE HEAT CONDUCTION}

An infinitely long cylindrical wall, made of a hydride forming metal, is considered. The wall contains hydrogen in solid solution as well as hydrides, which have precipitated within an annulus of the cross section, as shown in Fig. (1). The internal and external radii of the hydrided annulus are $r_{\text {in }}$ and $r_{e x}$, respectively, satisfying the relation: $r_{1} \leq r_{i n}<r_{e x} \leq r_{2}$, where $r_{1}$ and $r_{2}$ are internal and external radii of the cylindrical wall. There is no other restriction on the values of $r_{i n}$ and $r_{e x}$. Consequently, the hydride precipitation zone could extend through the whole thickness of the cylinder or it could occupy only an external or an internal layer of the cylinder, depending on the temperature distribution, the amount of hydrogen and the applied mechanical loading. In the case of a very thin cylindrical wall, $\left(r_{2}-r_{1}<<r_{1}\right)$, the geometry could be well approached by an infinite plate, shown in Fig. (2), with a hydride precipitation zone of thickness $L$.

The stress and temperature conditions on the internal and external boundary of the hydride precipitation annulus are the following:

$$
\begin{aligned}
& T=T_{i n}, \sigma_{k k}=\sigma_{m m}^{i n}, a t r=r_{i n} \\
& T=T_{e x}, a t r=r_{e x}
\end{aligned}
$$

$r$ is the radial coordinate of a cylindrical coordinate system, $(r, \theta, z)$, with $z$-axis coinciding with the axis of the cylindrical wall. When the hydride precipitation zone extends through the whole thickness of the cylindrical wall, the above boundary conditions are derived from prescribed wall surface temperatures and internal pressure. Additionally, it is 
assumed that the conditions of wall surfaces minimize the loss of hydrogen and allow the approach of zero hydrogen flux in the zone of hydride precipitation. For example wall surfaces, impermeable to hydrogen, satisfy the above assumption. In the case of zirconium nuclear fuel cladding, discussed in the following, the external oxide layer, which develops during reactor operation, is impermeable to hydrogen and, together with the negative radial temperature gradient, satisfies the above assumption.

Under steady state conditions for heat conduction and hydrogen diffusion, the governing equation of nonmechanical energy flow takes the following form:

$\frac{\partial}{\partial x_{i}}\left(k \frac{\partial T}{\partial x_{i}}\right)=0$

Relation (8) is obtained from relation (2.19) of [15], when the time-dependent terms of material heat content and heat released by hydride precipitation as well as the heat generated by hydrogen diffusion are set equal to zero. It is emphasized that even though relation (8) is not coupled to hydrogen diffusion, hydride precipitation and material deformation, the strong coupling between stress and temperature, in the hydride precipitation zone, is present and given by relations (4) and (5). Indeed, as steady state is approached, hydrogen flux approaches zero due to the opposite effects of non-zero thermal transport and stress-driven diffusion. Equally important, hydrogen concentration, in solid solution, approaches, throughout hydride precipitation zone, hydrogen terminal solid solubility, which strongly depends on temperature and stress. Relation (8) is identical to the well-known steady-state heat conduction governing equation (e.g. [22]). $k$ is the thermal conductivity of the metal, taken to be equal to the thermal conductivity of the hydride. Thermal conductivity generally depends on temperature, leading to non-linearity of (8). This characteristic is removed by the Kirchhoff transformation (e.g. [22, 23]):

$d B=\frac{k(T)}{k_{0}} d T$

where $k_{0}$ is the thermal conductivity at $T=T_{0}$. Then, the linear Laplace equation is derived:

$\frac{\partial}{\partial x_{i}}\left(\frac{\partial B}{\partial x_{i}}\right)=\nabla^{2} B=0$

which takes the following form, when the axial symmetry of the present problem is taken into account:

$\frac{d^{2} B}{d r^{2}}+\frac{1}{r} \frac{d B}{d r}=0$

Straightforward integration of (11), together with (9) and the boundary conditions (7a) and (7b), provides the distribution of $B$ and consequently of temperature, within the hydride precipitation zone of the cylindrical wall:

$$
\begin{aligned}
& B(T)=\int_{T_{0}}^{T} \frac{k(T)}{k_{0}} d T=\frac{1}{\ln \left(r_{e x} / r_{i n}\right)}\left[\left(B_{e x}-B_{i n}\right) \ln r\right. \\
& \left.+B_{i n} \ln r_{e x}-B_{e x} \ln r_{i n}\right]
\end{aligned}
$$

Table 1. Material properties of Zircaloy-2 and $\delta$-hydride

\begin{tabular}{|c|c|}
\hline$k$ & $9.37683+0.0118 T \mathrm{~W} \mathrm{~m}^{-1} \mathrm{~K}^{-1}$ \\
\hline$Q^{H}$ & $25122 \mathrm{~J} \mathrm{~mol}^{-1}$ \\
\hline$V^{H}$ & $7 \cdot 10^{-7} \mathrm{~m}^{3} \mathrm{~mol}^{-1}$ \\
\hline
\end{tabular}
$\left(\mathrm{ZrH}_{1.66}\right)$.

where $B_{e x}=B\left(T_{e x}\right)$ and $B_{i n}=B\left(T_{i n}\right)$.

Due to the axial symmetry of temperature distribution, the stress trace depends only on $r$. Substitution of (12) into (4) provides the radial, non-zero, component of stress trace gradient, within the hydride precipitation zone:

$\frac{d \sigma_{m m}}{d r}=\frac{3 Q^{H}}{\bar{V}^{H}} \frac{k_{0}}{k(T)} \frac{1}{\operatorname{Tr}}\left[\frac{B_{e x}-B_{i n}}{\ln \left(r_{e x} / r_{i n}\right)}\right]$

The general solution of the cylindrical wall problem is applied to the case of Zircaloy- 2 and $\delta$-hydride, with material properties given in Table 1. Zircaloy-2 is used for the construction of fuel cladding rods in light water nuclear reactors. During reactor operation, zirconium oxidation by coolant water, on the external rod surface, leads to the generation of hydrogen, which subsequently diffuses in the cladding. Hydride precipitation occurs, when sufficient hydrogen is produced to locally reach terminal solid solubility. In the present analysis, $r_{i n}$ and $r_{e x}$ are set equal to $5.325 \mathrm{~mm}$ and 6.125 $\mathrm{mm}$, respectively, which correspond to fuel cladding dimensions, examined in [15]. Also $T_{i n}$ and $T_{e x}$ are taken equal to $607 \mathrm{~K}$ and $567 \mathrm{~K}$, respectively. These temperatures are expected during reactor operation [15]. The temperature decreases across the cladding wall, as the heat, generated by nuclear fission within the nuclear fuel pellet, is removed by the coolant water, which surrounds the external surface of the fuel cladding.

The temperature and hydrostatic stress distributions within the cylindrical wall, made of Zircaloy-2, when hydride precipitation zone extends through the whole thickness of the wall and for the temperature boundary conditions, discussed above, are presented in Fig. (3). Hydrostatic stress has been normalized by $\sigma_{0}$, equal to $580 \mathrm{MPa}$, the irradiated Zircaloy-2 yield stress at $573 \mathrm{~K}$ [16]. Both distributions are nearly linear. Note that according to (5):

$\frac{\sigma_{k k}-\sigma_{m m}^{i n}}{3 \sigma_{0}}=\frac{Q^{H}}{\sigma_{0} \bar{V}^{H}} \ln \left(\frac{T}{T_{i n}}\right) \cong \frac{Q^{H}}{\sigma_{0} \bar{V}^{H}}\left(\frac{T-T_{i n}}{T_{i n}}\right)$

The approximate relation is derived by expanding $\ln \left(T / T_{\text {in }}\right)$ into a series and keeping the first significant term, under the condition $\left.-1<\left(T-T_{i n}\right)\right\} T_{i n} \leq 1$ (e.g. [24]). Therefore, according to (14), a nearly linear temperature distribution leads to a nearly linear hydrostatic stress distribution.

In the case of a very thin wall, when the geometry of the cylindrical wall is well approached by a plate, the distributions of temperature and stress trace gradient, within the hydride precipitation zone, are given by the following relations:

$$
B(T)=\int_{T_{0}}^{T} \frac{k(T)}{k_{0}} d T=\frac{B_{e x}-B_{i n}}{L} y+B_{i n},
$$



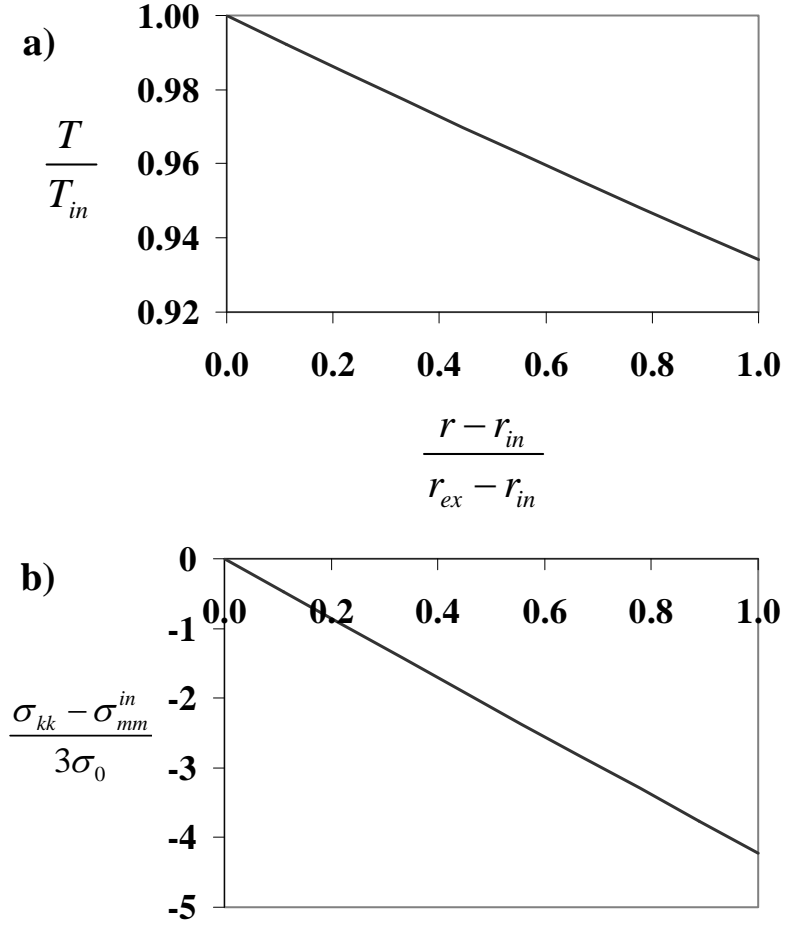

Fig. (3). Radial distribution of (a) temperature and (b) hydrostatic stress, within the hydride precipitation zone of a Zircaloy-2 cylinder, under steady-state hydrogen diffusion and heat conduction.

$$
\frac{d \sigma_{m m}}{d y}=\frac{3 Q^{H}}{\bar{V}^{H}} \frac{k_{0}}{k(T)} \frac{1}{T}\left(\frac{B_{e x}-B_{i n}}{L}\right)
$$

$y$ is the coordinate normal to the plate. Also $B_{i n}=B\left(T_{i n}\right)$ and $B_{e x}=B\left(T_{e x}\right) . T_{i n}$ and $T_{e x}$ are the temperatures on the sides of the hydride precipitation zone, where $y$ equals to 0 and $L$, respectively. The stress trace on $T_{i n}$-side of the hydride precipitation zone is taken equal to $\sigma_{m m}^{i n}$. Due to the applied boundary conditions, there is variation of temperature and stress trace, only perpendicularly to the plate.

Relation (12) is valid even in the presence of a radial crack on the external surface. Indeed, the radial geometrical discontinuity does not alter the conditions of heat conduction, which continues to show axial symmetry. The crack stress field initially attracts hydrogen and may lead to the development of a hydride precipitation zone ahead of the crack front, within only a sector of the cross section. Therefore the hydride precipitation zone does not present axial symmetry. However, after the redistribution of hydrogen and the development of steady state conditions, relations (4) and (5) are valid within the hydride precipitation zone. Consequently, the stress trace gradient, ahead of the crack within the hydride precipitation zone, has only a radial non-zero component, given by (13). Note that hydrostatic stress decreases as the crack tip is approached, against any prediction given by the well-known asymptotic crack-tip fields. Similarly, relations (15) and (16) are valid in the presence of a surface crack normal to the plate. Again the hydride precipitation zone may extend only ahead of the crack front. Rela- tion (14) is also valid in the presence of the surface cracks, discussed above; in these cases, $\sigma_{m m}^{i n}$ is the stress trace on the section of the boundary of the hydride precipitation zone, where $T$ equals $T_{\text {in }}$.

Relations (15) and (16) are applied to the case of a Zircaloy-2 plate of thickness equal to $0.8 \mathrm{~mm}$, under surface temperatures $T_{i n}$ and $T_{e x}$, equal to $607 \mathrm{~K}$ and $567 \mathrm{~K}$, respectively; this plate problem approaches the nuclear fuel cladding problem, considered previously. The temperature and stress trace distributions are again nearly linear. Also relation (16) provides a value of $\left(1 / \sigma_{0}\right) d \sigma_{m m} / d y$ equal to $-16.61 \mathrm{~mm}^{-1}$

at $T=567 \mathrm{~K}$. This value of stress trace gradient differs only by $1.63 \%$ from the value, calculated by the detailed finite element analysis in [15]. In the finite element analysis, the transient problem of coupled hydrogen diffusion, hydride precipitation and heat flow, under mechanical loading, was investigated in the presence of a surface crack normal to the plate on the cold side; this type of crack has been observed in experiments and it is generated by the fracture of the brittle oxide layer. The stress trace distributions of the finite element analysis are given in Fig. (13c) of [15]; it is clearly shown that the stress trace varies almost linearly, in the hydride precipitation zone, and it decreases, as the crack tip is approached. The $1.63 \%$ deviation is calculated between the prediction of relation (16) and the finite element distribution after 5-days of applied loading, when steady state conditions for hydrogen diffusion have been reached, according to Fig. (14) of [15]; heat conduction occurs also under steady state conditions.

\section{CONCLUSIONS}

In the present analysis, steady state for hydrogen diffusion, i.e. zero hydrogen flux, was considered, in a hydride forming metal. It was also assumed that hydride precipitation occurs under local chemical equilibrium among hydrogen, metal and hydride. The general stress conditions, which dominate in the zone of hydride precipitation, were then derived, by taking into account the coupling of the operating physical processes. According to these conditions, the stress trace is a logarithmic function of temperature. Consequently the stress trace is constant under constant temperature. The conditions are valid, irrespective of material elastic anisotropy, plastic deformation and geometry. Therefore they are valid, even when the hydride precipitation zone develops ahead of a crack tip. Thus the dominance of the well-known asymptotic crack-tip fields, including those of constraint based fracture mechanics, is lost in the area of hydride precipitation.

The general stress conditions were applied to the cases of a hydrided cylindrical wall and a hydrided plate, under steady state heat conduction, which present industrial interest. The analytical prediction on stress trace gradient, which is nearly a constant, is in excellent agreement with detailed finite element calculations. In the above cases, as well as in the case of constant temperature, hydrostatic stress, within the hydride precipitation zone, takes values, which are smaller than those, if the well-known asymptotic crack-tip stress fields were dominating. Indeed, hydride expansion during precipitation, in a zone ahead of a crack, leads to 
crack-tip shielding. However the fracture toughness of the material, in the hydride precipitation zone, consisting of solid solution and brittle hydrides, is significantly smaller than that of metal. Consequently the opposite effects of crack-tip shielding and material embrittlement need to be assessed.

Based on present analysis, it is concluded that fracture mechanics approaches, when employed for integrity assessments of structures under hydride-induced embrittlement, are applied rigorously and effectively, only following verification of asymptotic crack-tip field dominance, i.e. only when there is an annulus of dominance of the asymptotic crack-tip field, containing well within the zone of hydride precipitation.

In addition to above-mentioned implications on structural integrity assessments, the general stress conditions may prove to be important tools in multi-scale modeling (e.g. [2527]), where the texture of the hydride forming metal, the presence of phases with different hydrogen diffusion and solubility properties, as well as the hydride habit planes, within grains, could be considered.

\section{CONFLICT OF INTEREST}

The author confirms that this article content has no conflicts of interest.

\section{ACKNOWLEDGEMENT}

Declared none.

\section{REFERENCES}

[1] Williams ML. On the stress distribution at the base of a stationary crack. J Appl Mech 1957; 24: 109-14.

[2] Rice JR. A path independent integral and approximate analysis of strain concentration by notches and cracks. J Appl Mech 1968; 35: 379-86.

[3] Hutchinson JW. Singular behavior at the end of a tensile crack in a hardening material. J Mech Phys Solids 1968; 16: 13-31.

[4] Rice JR, Rosengren GF. Plane strain deformation near a crack tip in a power law hardening material. J Mech Phys Solids 1968; 16: $1-12$.

[5] Hutchinson JW. Fundamentals of the phenomenological theory of nonlinear fracture mechanics. J Appl Mech 1983; 50: 1042-51.

[6] Betegon C, Hancock JW. Two parameter characterization of elastic plastic crack tip fields. J Appl Mech 1991; 58: 104-10.
[7] O'Dowd NP, Shih CF. Family of crack tip fields characterized by a triaxiality parameter - I. Structure of fields. J Mech Phys Solids 1991; 39: 989-1015.

[8] Varias AG, Shih CF. Quasi-static crack advance under a range of constraints - Steady-state fields based on a characteristic length. J Mech Phys Solids 1993; 41: 835-61.

[9] Huang FH, Mills WJ. Delayed hydride cracking behavior for Zircaloy-2 tubing. Metall Trans A 1991; 22: 2049-60.

[10] Huang JH, Ho CS. Subcritical crack growth behavior for hydrided Zircaloy-4 plate. Mater Chem Phys 1997; 47: 184-92.

[11] Efsing P. Delayed Hydride Cracking in Irradiated Zircaloy. Stockholm: Royal Institute of Technology 1998.

[12] Yan D, Eadie RL. The near threshold behaviour of delayed hydride cracking in Zr-2.5 wt \% Nb. Int J Press Vessels Pip 2000; 77: 16777.

[13] Dutton R, Nuttall K, Puls MP, Simpson LA. Mechanisms of hydrogen induced delayed cracking in hydride forming materials. Metall Trans A 1977; 8: 1553-62.

[14] Sagat S, Chow CK, Puls MP, Coleman CE. Delayed hydride cracking in zirconium alloys in a temperature gradient. J Nucl Mater 2000; 279: 107-17.

[15] Varias AG, Massih AR. Hydride-induced embrittlement and fracture in metals - Effect of stress and temperature distribution. $\mathbf{J}$ Mech Phys Solids 2002; 50: 1469-510.

[16] Varias AG, Feng JL. Simulation of hydride-induced steady-state crack growth in metals - Part I: growth near hydrogen chemical equilibrium. Comput Mech 2004; 34: 339-56.

[17] Varias AG, Feng JL. Simulation of hydride-induced steady-state crack growth in metals - Part II: general near tip field. Comput Mech 2004; 34: 357-76.

[18] Northwood DO, Kosasih U. Hydrides and delayed hydrogen cracking in zirconium and its alloys. Int Met Rev 1983; 28: 92-121.

[19] Shewmon PG. Diffusion in Solids. The Minerals, Metals \& Materials Society: Warrendale, Pennsylvania; 1989.

[20] Li JCM, Oriani RA, Darken LS. The thermodynamics of stressed solids. Z Phys Chem (N F) 1966; 49: 271-90.

[21] Denbigh KG. The Thermodynamics of the Steady State. London: Methuen 1951.

[22] Gebhart B. Heat Transfer. New York: McGraw-Hill 1971.

[23] Ames WF. Nonlinear partial differential equations in engineering. New York: Academic Press 1965.

[24] Gradshteyn IS, Ryzhik IM. Tables of Integrals, Series, and Products. London: Academic Press; 1980.

[25] Kalweit M, Drikakis D. Multiscale methods for micro/nano flows and materials. J Comput Theor Nanosci 2008; 5: 1923-38.

[26] Samaras M, Victoria M, Hoffelner W. Nuclear energy materials prediction: application of the multi-scale modeling paradigm. Nucl Eng Technol 2009; 41: 1-10.

[27] Ghosh S, Lee K, Raghavan P. A multi-level computational model for multi-scale damage analysis in composite and porous materials. Int J Solids Struct 2001; 38: 2335-85.

(C) A.G. Varias; Licensee Bentham Open.

This is an open access article licensed under the terms of the Creative Commons Attribution Non-Commercial License (http://creativecommons.org/licenses/by-nc/3.0/) which permits unrestricted, non-commercial use, distribution and reproduction in any medium, provided the work is properly cited. 